\title{
Cooperative Beamforming and Jamming for Secure VLC System in the Presence of Active and Passive Eavesdroppers
}

\author{
Sunghwan Cho, Member, IEEE, Gaojie Chen, Senior Member, IEEE, and Justin P. Coon, Senior Member, IEEE
}

\begin{abstract}
This paper proposes a novel cooperative beamforming and jamming scheme to deal with passive and active eavesdroppers (EDs) in indoor visible light communication (VLC) networks. An ED in VLC systems can augment its front-end receiver by implementing possible device modifications; thus, jamming is very useful for curbing such an enhanced ED since it would be impossible to distinguish between the information and jamming signals. In contrast to the traditional artificial noise strategies for VLC that can only deal with either passive or active EDs, we propose a combined scheme of beamforming and jamming that significantly improves secrecy performance when both types of EDs exist. The proposed scheme is designed to maximize the signal-to-interference-plus-noise ratio (SINR) of the legitimate receiver, entirely suppress the SINRs of the active EDs, and restrict the average SINR of the passive EDs. We apply an inverse free preconditioned Krylov subspace projection method and the convex-concave procedure to obtain the suboptimal beamforming weight and jamming intensity vectors. Also, an optimal power splitter coefficient is found through the golden section search method. The numerical results verify that the proposed scheme shows superior performance compared to the three benchmarks: zero-forcing beamforming, artificial noise scheme, and enhanced zero-forcing beamforming.
\end{abstract}

Index Terms-Visible light communication, physical layer security, cooperative beamforming and jamming, eavesdropper.

\section{INTRODUCTION}

The acceleration of mobile devices' development, such as smartphones, tablet computers, Internet of Things (IoT) devices, wearable devices, etc., has provoked a higher demand for data traffic via wireless communication. However, conventional radio frequency (RF) communication networks, for example, Wireless Fidelity (WiFi) and cellular networks, face difficulties in satisfying future networks' requirements due to the RF spectrum's scarcity. Moreover, the rapidly increasing data traffic would necessitate the expansion of communication infrastructure, which in turn will lead to an enormous increase in energy usage. As a potential solution, visible light communication (VLC) has attained considerable popularity in academia and industry. VLC can offer significantly greater capacity due to the broad spectrum ranging from $400 \mathrm{THz}$

The work of Gaojie Chen was supported by the EPSRC under Grant EP/R006377/1 ("M3NETs").

S. Cho is with the Department of Electrical Engineering, Korea Military Academy, Seoul, 01809, South Korea (e-mail: scho6463@gmail.com or scho@kma.ac.kr).

J. P. Coon is with the Department of Engineering Science, University of Oxford, Oxford, OX1 3PJ, U.K. (e-mail: justin.coon@eng.ox.ac.uk).

G. Chen is with the School of Engineering, University of Leicester, Leicester, LE1 7RH, U.K. (e-mail: gaojie.chen@leicester.ac.uk). to $700 \mathrm{THz}$; high spectral efficiency due to the unlicensed spectrum; and low energy consumption and infrastructure cost, since VLC systems use the existing lighting infrastructure for both illumination and communications purposes. Hence, VLC systems can be an excellent possible solution to support future networks, allowing high transmission rates, ultralow latency, extensive device connectivity, greater energy efficiency, lower traffic cost, etc. [1], [2].

Furthermore, visible light cannot penetrate opaque materials; thus, the VLC networks provide high security at the physical layer. However, in large open spaces, namely, libraries, open-plan offices, and conference halls, there is still a risk that malicious users may eavesdrop on transmitted signals in VLC systems. Although an authentication process similar to $\mathrm{WiFi}$ Protected Access (WPA) would be possible even for a VLC system, it was shown that this method might be breakable [3]; thus, it would not be entirely secure.

Among many network security approaches, physical layer security (PLS) is a set of techniques that utilize channel randomness to allow the transmitter and intended receiver to exchange important data [4]. PLS can be deemed the most secure communication method since security in this context is provable (in the information-theoretic sense). Spurred by the advantages of PLS, various PLS methods tailored for VLC environments have been proposed and investigated. An extensive research of this was first made by Mostafa et al. [5]-[9]. More specifically, in [5], expressions for achievable secrecy rates were given for single-input single-output (SISO) and multiple-input single-output (MISO) systems, and a robust beamforming technique was proposed. Another extensive series of studies has been conducted by Alouini et al. [10][12]. In [10], [11], secrecy rates were investigated with several input distributions under the amplitude constraint, for example, truncated generalized normal distribution, truncated Gaussian distribution, and uniform distribution. Also, PLS for a spatial modulation-based VLC system has been studied [12]. Besides, Pham et al. proposed novel ZF and artificial noiseaided precoding schemes, respectively, to enhance the PLS for multiuser MISO VLC networks [13]-[15]. More specifically, [13] employed a ZF precoder to maximize the secrecy sumrate, and [14] proposed an optimal artificial noise-assisted precoding algorithm to enhance the SINR of the UE with ensuring the fairness of the UE's SINRs and disturbing the information reception of ED. Also, [15] designed artificial noiseaided precoding to minimize the total transmit power and improve energy efficiency. In addition, [16] studied the effect 
of both the specular and the diffusive reflections by proposing a modified Monte Carlo ray-tracing model. Moreover, the VLC system with simultaneous lightwave information and power transfer (SLIPT) was investigated for the scenario in which both information receiver and energy receiver are present [17]. More recent studies on the PLS in VLC networks can be found in [18] and [19].

On the one hand, considering the channel model of VLC systems given in [20], an ED can significantly enhance its receiver capability by implementing possible device modifications, such as increasing the photodiode's (PD's) physical area, utilizing a high-gain optical lens, and precisely aligning the receiver's orientation toward the information LED transmitter. For instance, [21] utilized a telescope to enhance the receiver gain in an experimental test and showed that the VLC transmission could be successfully eavesdropped at a considerable distance from the transmitter. The legitimate user (UE) also can enhance its receiver sensitivity using these methods. However, the UE must consider the other constraints on hardware requirements, user convenience, and communication efficiency for a given application scenario, which limits the gain of the UE. On the other hand, EDs can ignore these issues.

To cope with such an augmented ED, various artificial jamming strategies for VLC systems have been proposed [6], [8], [10], [22], [23]. The jamming strategy is a practical and robust approach, particularly against EDs with enhanced receiver architectures, since it would be impossible for an offending device to distinguish between information and jamming signals. On the other hand, existing jamming strategies consider either passive or active EDs, but not both. Here, the passive and active EDs are different in the availability of the channel state information (CSI) and/or the location information of the EDs at the transmitters. In practice, EDs may attempt to evade a legitimate user's vigilance; thus, it would be challenging to know the (passive) EDs' locations, types, and numbers. In this sense, it would be crucial to consider both active and passive EDs in designing jamming schemes. Besides, our recent work [24] proposed a zeroforcing (ZF) beamforming method, which could cope with both passive and active EDs simultaneously, but it did not consider jamming approaches; thus, it might be vulnerable to the augmented EDs ${ }^{1}$.

This paper will propose a combined beamforming and jamming scheme that copes with active and passive EDs simultaneously. This work is the first to utilize an artificial noise approach to prevent both types of EDs from wiretapping information in VLC systems. The proposed scheme will maximize the SINR of the UE while entirely suppressing the SINRs of the active EDs (AEDs) and restraining the average SINR of the passive EDs (PEDs). We consider the AEDs as more apparent security threats than PEDs; therefore, we apply a stricter constraint to the AEDs than to the PEDs. We also utilize tools from stochastic geometry (similar to [22], [23], [25]) to model the random locations of PEDs.

We formulate an optimization problem to obtain solutions for beamforming and jamming vectors and a power

\footnotetext{
${ }^{1}$ In Section IV, we will explain an example for this case.
}

splitter coefficient. However, since this original problem is intractable, we separate this into two different optimization problems related to beamforming and jamming transmissions, respectively. Concerning the jamming part, we find a jamming vector that lies in the UE's channel's null space not to cause interference at the UE's site. Besides, concerning the beamforming (information transmission) part, we make a beamforming vector to lie in the AEDs' channel matrix's null space so that the AEDs do not receive any information signals. Also, to maximize the received signal power at the UE's site, we direct the information beam with a particular eigenmode associated with the UE's channel matrix and the passive ED's intensity. We find the eigenmode by employing an inverse free preconditioned Krylov subspace projection method. Lastly, the optimal power splitter coefficient, which yields the maximum SINR at the UE, is numerically found through a golden section search (GSS) method.

The rest of this paper is organized as follows. The system model, including the room configuration and the VLC channel model, is provided in Section II. In Section III, the optimization problem for the proposed scheme is designed and solved. Section IV provides numerical and simulation results to verify our analysis. The conclusion of this paper will be given in Section V.

\section{SySTEM MODEL}

\section{A. Room Configuration}

Fig. 1 illustrates the considered VLC system consisting of $N$ LED transmitters attached to the ceiling of the room, where $H$ represents the height of a ceiling relative to the work plane, and $L$ and $W$ indicate the length and the width of the room, respectively. We assume that each LED transmitter - an LED fixture consisting of multiple individual LEDs - broadcasts a light signal independent of the signals sent by other transmitters. Besides, one UE, $N_{A}$ AEDs, and multiple PEDs are assumed to be present in the (grey) work sphere. Also, it is assumed that the exact location information and/or CSI of the UE and AEDs are available at the transmitter; in contrast, for the PEDs, only their statistical location information is available. That is, we assume that the locations of PED are randomly and uniformly distributed according to a homogeneous Poisson Point Process (PPP) $\Phi_{P E}$ with density $\lambda_{P E}$ [26]. Notice that the uniform distribution of PEDs signifies that the locations of PEDs are entirely random, which can be regarded as the worst case from the security perspective [27]. The UE, the AEDs, and the PEDs are represented by $U E, A E_{p}$ for $p \in\left\{1,2, \ldots, N_{A}\right\}$, and $P E_{q}$ for $q \in\{1,2, \ldots\}$, respectively. Also, we assume that there is no collusion among EDs.

\section{B. Data Transmission and Jamming}

To improve the security of VLC networks, a combined method of beamforming and jamming is utilized. The information signal $x(t) \in[-1,1]$ is created from a certain real constellation and multiplied by a beamforming weight $\omega_{i}$ $\left(\left|\omega_{i}\right| \leq 1\right)$ at the $i$ th transmitter for $i \in\{1,2, \ldots, N\}$. Besides, the $i$ th transmitter can transmit a random jamming interference $j(t) \in[-1,1]$ after multiplying a jamming intensity weight $v_{i}$ 


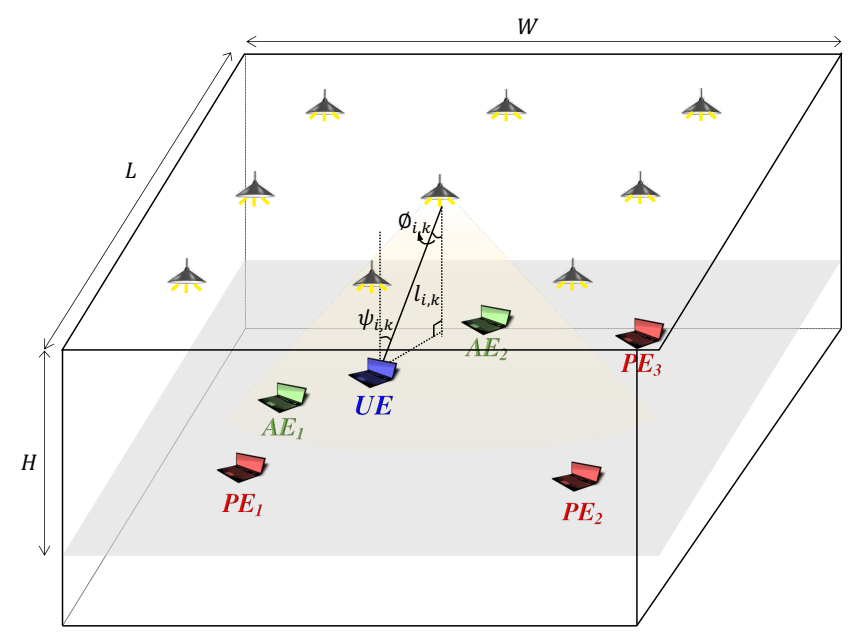

Fig. 1: Rectangular room configuration for VLC systems.

$\left(\left|v_{i}\right| \leq 1\right)$. Note that in the considered MISO beamforming and jamming scheme, the data and jamming signals $x(t)$ and $j(t)$ are assumed to be identical for all the transmitters as in [22]. Therefore, the input signal at the $i$ th transmitter can be written as

$$
s_{i}(t)=\alpha I_{D C}\left(\rho \omega_{i} x(t)+(1-\rho) v_{i} j(t)\right)
$$

where $\alpha \in[0,1]$ denotes the modulation index and $I_{D C} \in$ $\mathbb{R}_{+}$is a fixed bias current. Here, $\mathbb{R}_{+}$denotes the set of nonnegative real numbers. $\rho \in[0,1]$ denotes the power splitter coefficient for an information signal, while $1-\rho$ is that of a jamming signal. The input signal $s_{i}(t)$ is superimposed on a fixed bias current $I_{D C}$, which is used for illumination. Also, we constrain the amplitude of $s_{i}(t)$ such that $\left|s_{i}(t)\right| \leq \alpha I_{D C}$ to maintain linear current-to-light conversion. Therefore, the dynamic range of the LED is $I_{D C} \pm \alpha I_{D C}$. Also, $\mathbb{E}\left[s_{i}(t)\right]=0$ is assumed; thus, the data and jamming signals do not affect the level of illumination.

According to [20], the channel gain $h_{i, k} \in \mathbb{R}_{+}$from the $i$ th transmitter to the receiver $k \in\left\{U E, A E_{p}, P E_{q}\right\}$ corresponding to an LED with a generalized Lambertian emission pattern in a VLC system is given by $h_{i, k}=\eta G_{i, k} T_{k} R_{k}$, where $\eta$ (W/A) is the current-to-light conversion efficiency, $T_{k}$ (V/A) is the transimpedance amplifier gain, and $R_{k}(\mathrm{~mA} / \mathrm{mW})$ is the photodetector's responsivity. Also, $G_{i, k}$ signifies the Line-ofSight (LoS) path gain, which is expressed as

$$
\begin{aligned}
& G_{i, k}= \\
& \left\{\begin{array}{ccc}
\frac{(m+1) A_{\mathrm{PD}, k}}{2 \pi l_{i, k}^{2}} \frac{\kappa_{k}^{2} \cos ^{m}\left(\phi_{i, k}\right)}{\sin ^{2}\left(\Psi_{c}\right)} \cos \left(\psi_{i, k}\right) & \text { for } & \left|\psi_{i, k}\right| \leq \Psi_{c}, \\
0 & \text { for } & \left|\psi_{i, k}\right|>\Psi_{c}
\end{array}\right.
\end{aligned}
$$

where $m=-\ln (2) / \ln \left(\cos \left(\phi_{1 / 2}\right)\right)$ is the order of Lambertian emission with half illuminance at $\phi_{1 / 2}$, and $A_{\mathrm{PD}, k}\left(\mathrm{~m}^{2}\right)$ is the reciever PD's physical area. As depicted in Fig. $1, l_{i, k}$ (m) denotes the distance between the $i$ th transmitter and the receiver $k . \psi_{i, k}$ and $\phi_{i, k}$ denotes the angles of incidence and irradiance, respectively. $\kappa_{k}$ is the refractive index of the optical concentrator, and $\Psi_{c}$ denotes the PD's received field of view. With assuming that a receiver's PD faces up perpendicular to the work plane, i.e., assuming $\phi_{i, k}=\psi_{i, k}$, the channel gain can be rewritten as $h_{i, k}=K l_{i, k}^{-(m+3)}$, where $K=\left(\eta(m+1) A_{\mathrm{PD}, k} H^{m+1} \kappa_{k}^{2} R_{k} T_{k}\right) /\left(2 \pi \sin ^{2}\left(\Psi_{c}\right)\right)$. This assumption may yield a gap between theoretical and experimental channel gains when the orientation of the receiver's PD is not perpendicular. Indeed, this would be a typical case in reality. However, note that the proposed schemes would be implemented in reality by using the CSI of the UE and the AEDs measured by the channel estimation techniques, e.g., using a pilot [28], [29], so that the various imperfections of the theoretical VLC channel model can be excluded. In contrast, the PEDs' channels still need to be stochastically modeled with the proposed mathematical model in (2) based on their unknown locations.

Hence, the received signal at receiver $k$ under the cooperative beamforming and jamming scheme can be written as

$$
y_{k}(t)=\alpha I_{D C} \mathbf{h}_{k}^{T}(\rho \mathbf{w} x(t)+(1-\rho) \mathbf{v} j(t))+n_{k}(t)
$$

where $\mathbf{h}_{k}=\left[h_{1, k}, h_{2, k}, \ldots, h_{N, k}\right]^{T} \in \mathbb{R}^{N}$ is the channel gain vector, and $\mathbf{w}=\left[\omega_{1}, \omega_{2}, \ldots, \omega_{N}\right]^{T}$ and $\mathbf{v}=\left[v_{1}, v_{2}, \ldots, v_{N}\right]^{T}$ represent the beamforming weight and jamming intensity vectors, respectively. $\mathbb{R}$ denotes the set of real numbers. Also, $n_{k}(t)$ denotes zero-mean additive white Gaussian noise (AWGN) at receiver $k$ with variance $\sigma^{2}$. For notational convenience, we drop the time index $t$ for the remainder of the paper.

\section{Performance Metrics}

As similar to [8], [22], we consider a Gaussian VLC MISO channel with an amplitude constraint and define the peak SINR by assuming $x=1$ and $j=1$. The peak SINR at receiver $k$ can be written as

$$
\gamma_{k}=\frac{\alpha^{2} I_{D C}^{2} \rho^{2} \mathbf{w}^{T} \mathbf{H}_{k} \mathbf{w}}{\alpha^{2} I_{D C}^{2}(1-\rho)^{2} \mathbf{v}^{T} \mathbf{H}_{k} \mathbf{v}+\sigma^{2}}=\frac{\mathcal{D}_{k}}{\mathcal{J}_{k}+\sigma^{2}}
$$

where $\mathbf{H}_{k}=\mathbf{h}_{k} \mathbf{h}_{k}^{T}$. Also, $\mathcal{D}_{k}=\alpha^{2} I_{D C}^{2} \rho^{2} \mathbf{w}^{T} \mathbf{H}_{k} \mathbf{w}$ and $\mathcal{J}_{k}=$ $\alpha^{2} I_{D C}^{2}(1-\rho)^{2} \mathbf{v}^{T} \mathbf{H}_{k} \mathbf{v}$ denote the received data power and the jamming interference power, respectively.

Also, as in [30], the secrecy outage probability (SOP) is defined as the probability that the SINR of UE is lower than the highest SINR of EDs, which can be given by

$$
P_{S O}=\mathbb{P}\left(\gamma_{U E}<\max _{q \in \Phi_{P E}} \gamma_{P E_{q}}\right) \stackrel{(a)}{=} 1-\exp \left(-\iint_{\mathcal{A}} \lambda_{P E} \mathrm{~d} \mathbf{x}\right)
$$

where $\mathbb{P}(\cdot)$ denotes the probability operator. The equality (a) holds due to the distribution of the contact distance to the nearest point of the PPP [26], where $\mathcal{A}=\{\mathbf{x} \mid \gamma(\mathbf{x}, \mathbf{w}, \mathbf{v})>$ $\left.\gamma_{U E}\right\}$ represents the region where the SINR is higher than the SINR of UE. Also, $\gamma(\mathbf{x}, \mathbf{w}, \mathbf{v})$ indicates the SINR measured at the point $\mathbf{x} \in \mathbb{R}^{2}$ in the work plane ${ }^{2}$, which can be described

${ }^{2} \mathrm{We}$ assume that the center of the room is the origin of the coordinate system. 
as

$$
\gamma(\mathbf{x}, \mathbf{w}, \mathbf{v})=\frac{\alpha^{2} I_{D C}^{2} \rho^{2} \mathbf{w}^{T} \mathbf{h}(\mathbf{x}) \mathbf{h}(\mathbf{x})^{T} \mathbf{w}}{\alpha^{2} I_{D C}^{2}(1-\rho)^{2} \mathbf{v}^{T} \mathbf{h}(\mathbf{x}) \mathbf{h}(\mathbf{x})^{T} \mathbf{v}+\sigma^{2}}
$$

where $\mathbf{h}(\mathbf{x})$ signifies the channel gain vector at the point $\mathbf{x}$.

\section{COOPERATIVE BEAMFORMING AND JAMMING Against ACtive AND PAssive EAVEsdroppers}

This section proposes a cooperative beamforming and jamming design by formulating an optimization problem that aims to improve secrecy performance in the presence of both the AEDs and PEDs.

The proposed optimization problem can be formulated as

$$
\begin{aligned}
& \left\{\rho^{\star}, \mathbf{w}^{\star}, \mathbf{v}^{\star}\right\}=\arg \max _{\rho, \mathbf{w}, \mathbf{v}} \gamma_{U E} \\
& \text { s.t. }\left\{\begin{array}{l}
\gamma_{A E_{p}}=0, \quad \forall p \in\left\{1,2, \ldots, N_{A}\right\} \\
\mathbb{E}\left[\gamma_{P E_{q}}\right] \leq \xi_{P E} \\
|\mathbf{w}| \leq \mathbb{1} \\
|\mathbf{v}| \leq \mathbb{1} \\
0 \leq \rho \leq 1
\end{array}\right.
\end{aligned}
$$

where $\mathbb{E}[\cdot]$ denotes the expectation operator, and $\mathbb{1}$ denotes the all-ones column vector. The objective is to maximize the SINR of UE, while the first constraint is to suppress the AEDs' SINRs to zero, and the second is to restrict the average SINR of a single PED to $\xi_{P E}$.

This work assumes that PED locations' knowledge is not available at the transmitter sites. Therefore, it would be natural to consider the average SINR of a single ${ }^{3}$ PED. However, since the second constraint does not lead to a tractable analysis, in a similar manner used in [22], [31], we replace the expectation of a single PED' SINR with the ratio of the average received data power to the average interference plus noise power as

$$
\begin{aligned}
\bar{\gamma}_{P E} & \triangleq \frac{\mathbb{E}\left[\alpha^{2} I_{D C}^{2} \rho^{2} \mathbf{w}^{T} \mathbf{H}_{P E_{q}} \mathbf{w}\right]}{\mathbb{E}\left[\alpha^{2} I_{D C}^{2}(1-\rho)^{2} \mathbf{v}^{T} \mathbf{H}_{P E_{q}} \mathbf{v}+\sigma^{2}\right]} \\
& =\frac{\alpha^{2} I_{D C}^{2} \rho^{2} \mathbf{w}^{T} \overline{\mathbf{H}}_{P E} \mathbf{w}}{\alpha^{2} I_{D C}^{2}(1-\rho)^{2} \mathbf{v}^{T} \overline{\mathbf{H}}_{P E} \mathbf{v}+\sigma^{2}} \\
& =\frac{\overline{\mathcal{D}}_{P E}}{\overline{\mathcal{J}}_{P E}+\sigma^{2}}
\end{aligned}
$$

where $\overline{\mathbf{H}}_{P E}=\mathbb{E}\left[\mathbf{h}_{P E_{q}} \mathbf{h}_{P E_{q}}^{T}\right]$ and its element can be obtained by

$$
\begin{aligned}
\bar{H}_{P E_{i, j}} & =\mathbb{E}\left[h_{i, P E_{q}} h_{j, P E_{q}}\right] \\
& =\mathbb{E}\left[K^{2} l_{i, P E_{q}}^{-(m+3)} l_{j, P E_{q}}^{-(m+3)}\right] \\
& =\frac{1}{L W} \int_{\frac{-L}{2}}^{\frac{L}{2}} \int_{\frac{-W}{2}}^{\frac{W}{2}} \frac{K^{2}}{l_{i}^{m+3}(\mathbf{x}) l_{j}^{m+3}(\mathbf{x})} \mathrm{d} \mathbf{x}
\end{aligned}
$$

where $l_{i}(\mathbf{x})=\sqrt{\left|\mathbf{x}-\mathbf{x}_{i}^{\mathrm{LED}}\right|^{2}+H^{2}}$ represents the distance between the $i$ th LED transmitter and the point $\mathbf{x} \in \mathbb{R}^{2}$, and $\mathbf{x}_{i}^{\mathrm{LED}} \in \mathbb{R}^{2}$ for $i \in\{1,2, \ldots, N\}$ is the location of the $i$ th

${ }^{3}$ Note that since no collusion among EDs is assumed, considering the average SINR of a single PED, rather than considering the SINR sum for multiple PEDs, would be more straightforward to compare with the SINR of the UE.
LED transmitter projected to the work plane. Also, $\overline{\mathcal{D}}_{P E}=$ $\alpha^{2} I_{D C}^{2} \rho^{2} \mathbf{w}^{T} \overline{\mathbf{H}}_{P E} \mathbf{w}$ and $\overline{\mathcal{J}}_{P E}=\alpha^{2} I_{D C}^{2}(1-\rho)^{2} \mathbf{v}^{T} \overline{\mathbf{H}}_{P E} \mathbf{v}$ denote the average received information and interference power of a PED, respectively. Although $\bar{\gamma}_{P E}$ does not approximate $\mathbb{E}\left[\gamma_{P E_{q}}\right]$, as we will see in Section IV, $\bar{\gamma}_{P E}$ is a useful metric for constraining the information reception and enhancing the interference in areas other than the UE location, while significantly reducing the optimization problem's complexities.

Now, the proposed optimization problem can be rewritten as

$$
\begin{aligned}
& \left\{\rho^{\star}, \mathbf{w}^{\star}, \mathbf{v}^{\star}\right\}=\underset{\rho, \mathbf{w}, \mathbf{v}}{\arg \max _{U E}} \mathcal{D}_{U E} \\
& \text { s.t. }\left\{\begin{array}{l}
\mathcal{J}_{U E}=0 \\
\mathcal{D}_{A E_{p}}=0, \quad \forall p \in\left\{1,2, \ldots, N_{A}\right\} \\
\overline{\mathcal{D}}_{P E} \leq \bar{\xi}_{P E} \\
\overline{\overline{\mathcal{J}}}_{P E}+\sigma^{2} \\
|\mathbf{w}| \leq \mathbb{1} \\
|\mathbf{v}| \leq \mathbb{1} \\
0 \leq \rho \leq 1
\end{array}\right.
\end{aligned}
$$

where $\mathcal{D}_{U E}=\alpha^{2} I_{D C}^{2} \rho^{2} \mathbf{w}^{T} \mathbf{H}_{U E} \mathbf{w}$ represents the received data power at the UE, and $\bar{\xi}_{P E}$ is the target constraint on $\bar{\gamma}_{P E}$. To facilitate solving the optimization problem (10), we separate this problem into two subordinate optimization problems, whose optimization variables are $\mathbf{v}$ and $\mathbf{w}$, respectively, by fixing the power splitter coefficient $\rho$ as below:

$$
\begin{gathered}
\mathcal{P} 1: \mathbf{v}^{*}=\arg \max _{\mathbf{v}} \overline{\mathcal{J}}_{P E} \\
\text { s.t. }\left\{\begin{array}{l}
\mathcal{J}_{U E}=0 \\
|\mathbf{v}| \leq \mathbb{1}
\end{array}\right. \\
\mathcal{P} 2: \mathbf{w}^{*}=\arg \max _{\mathbf{w}} \mathcal{D}_{U E} \\
\text { s.t. }\left\{\begin{array}{l}
\mathcal{D}_{A E_{p}}=0, \quad \forall p \in\left\{1,2, \ldots, N_{A}\right\} \\
\overline{\mathcal{D}}_{P E} \leq \bar{\xi}_{P E}\left(\overline{\mathcal{J}}_{P E}+\sigma^{2}\right) \\
|\mathbf{w}| \leq \mathbb{1} .
\end{array}\right.
\end{gathered}
$$

Finding the solution $\left\{\rho^{\star}, \mathbf{w}^{\star}, \mathbf{v}^{\star}\right\}$ of the original problem (7) comprises three steps. First, given a value of $\rho$, we solve $\mathcal{P} 1$ to obtain the suboptimal jamming vector $\mathbf{v}^{*}$ and its corresponding objective value $\overline{\mathcal{J}}_{P E}^{*}$. Second, we plug $\overline{\mathcal{J}}_{P E}^{*}$ into $\overline{\mathcal{J}}_{P E}$ in the right term of the second constraint in (12b) and solve $\mathcal{P} 2$ to obtain the beamformer $\mathbf{w}^{*}$. Third, we repeat the first and second steps varying the value of $\rho$ until the suboptimal SINR of the UE $\gamma_{U E}^{*}$ calculated by using $\mathbf{w}^{*}$ and $\mathbf{v}^{*}$ is maximized. The maximum value of $\gamma_{U E}^{*}$ over $\rho \in[0,1]$ is denoted by $\gamma_{U E}^{\star}$. Lastly, we set the final solution $\left\{\rho^{\star}, \mathbf{w}^{\star}, \mathbf{v}^{\star}\right\}$ to $\left\{\rho, \mathbf{w}^{*}, \mathbf{v}^{*}\right\}$ yielding $\gamma_{U E}^{\star}$. Note that although the final solution obtained from solving these separated problems $\mathcal{P} 1$ and $\mathcal{P} 2$ may not be identical to the solution of (10), separating (10) into $\mathcal{P} 1$ and $\mathcal{P} 2$ is a reasonable approach in a sense that $\overline{\mathcal{J}}_{P E}^{*}$ obtained from $\mathcal{P} 1$ expands the feasible space of $\mathbf{w}^{*}$ in $\mathcal{P} 2$ the most. In the following subsections, we provide solutions for each step. 


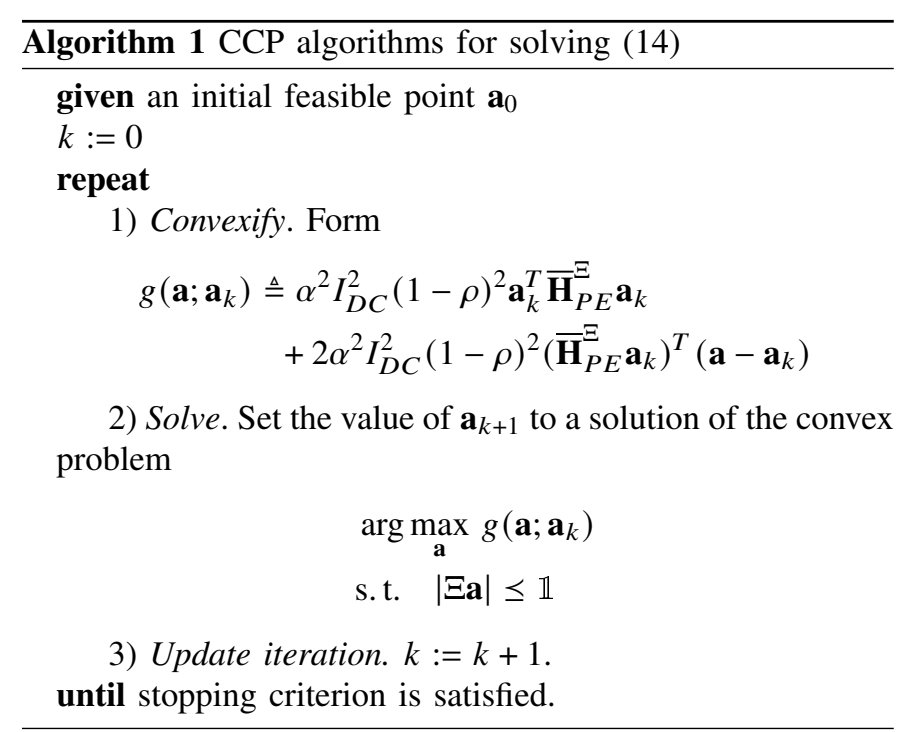

\section{A. Step 1: Solve $\mathcal{P} 1$}

To satisfy the first constraint in (11b), we restrain the jamming vector $\mathbf{v}$ to lie in the null space of the UE's channel vector $\mathbf{h}_{U E}$, which can be represented as

$$
\mathbf{v}=\Psi_{U E} \Psi_{U E}^{T} \mathbf{a}
$$

where $\Psi_{U E} \in \mathbb{R}^{N \times(N-1)}$ is a matrix whose $N-1$ columns constitute a basis for the null space of $\mathbf{h}_{U E}$, and $\mathbf{a} \in \mathbb{R}^{N}$ is an arbitrary real vector. Thus, by defining $\Xi=\Psi_{U E} \Psi_{U E}^{T}$, i.e., $\mathbf{v}=\Xi \mathbf{a}$, we restate the optimization problem (11) in terms of a as

$$
\begin{aligned}
\mathbf{a}^{*}= & \arg \max _{\mathbf{a}} \alpha^{2} I_{D C}^{2}(1-\rho)^{2} \mathbf{a}^{T} \overline{\mathbf{H}}_{P E}^{\Xi} \mathbf{a} \\
& \text { s.t. }|\Xi \mathbf{a}| \leq \mathbb{1}
\end{aligned}
$$

where $\overline{\mathbf{H}}_{P E}^{\Xi}=\Xi^{T} \overline{\mathbf{H}}_{P E} \Xi$. Here, analytically solving (14) is challenging since it needs to maximize a convex quadratic objective function with linear inequality constraints, which is a classical and challenging problem [32]. Thus, we apply the convex-concave procedure (CCP) method [33], which is an efficient heuristic approach to obtain a local solution.

The CCP method transforms the non-convex objective function into an affine (i.e., convex) function with a given initial feasible point $\mathbf{a}_{0}$ by employing the first-order Taylor series approximation, and we can solve the convex problem by using conventional optimization programmings, such as sequential quadratic programming (SQP) [34] and interiorpoint methods algorithm [35]. After setting the solution to $\mathbf{a}_{1}$, we iterate the same procedure until the objective value does not improve more than a predefined threshold $\epsilon_{c}$. Then, we can obtain the suboptimal jamming intensity vector from $\mathbf{v}^{*}=\Xi \mathbf{a}^{*}$. Algorithm 1 explains the details of the iterative CCP algorithm.

\section{B. Step 2: Solve $\mathcal{P} 2$}

Similarly to (13), to prevent the AEDs from receiving information signals, we constrain the beamforming vector $\mathbf{w}$ (related to an information transmission) to lie in the null space of the AEDs' channel matrix $\left[\begin{array}{lllll}\mathbf{h}_{A E_{1}} & \mathbf{h}_{A E_{2}} & \ldots & \mathbf{h}_{A E_{N_{A}}}\end{array}\right]^{T}$. This condition can be expressed as

$$
\mathbf{w}=\Psi_{A E} \Psi_{A E}^{T} \mathbf{b}
$$

where $\mathbf{b} \in \mathbb{R}^{N}$ is an arbitrary real vector and $\Psi_{A E} \in$ $\mathbb{R}^{N \times\left(N-N_{A}\right)}$ is a matrix whose $N-N_{A}$ columns constitute a basis for the null space. The condition (15) makes the first constraint of (12b) satisfied. Therefore, letting $\Theta=\Psi_{A E} \Psi_{A E}^{T}$, i.e., $\mathbf{w}=\Theta \mathbf{b}$, the optimization problem involving the vector $\mathbf{b}$ can be given as

$$
\begin{array}{r}
\mathbf{b}^{*}=\arg \max _{\mathbf{b}} \alpha^{2} I_{D C}^{2} \rho^{2} \mathbf{b}^{T} \mathbf{H}_{U E}^{\Theta} \mathbf{b} \\
\text { s.t. }\left\{\begin{array}{l}
\alpha^{2} I_{D C}^{2} \rho^{2} \mathbf{b}^{T} \overline{\mathbf{H}}_{P E}^{\Theta} \mathbf{b} \leq C \\
|\Theta \mathbf{b}| \leq \mathbb{1}
\end{array}\right.
\end{array}
$$

where $\mathbf{H}_{U E}^{\Theta}=\Theta^{T} \mathbf{H}_{U E} \Theta, \overline{\mathbf{H}}_{P E}^{\Theta}=\Theta^{T} \overline{\mathbf{H}}_{P E} \Theta$, and $C=$ $\bar{\xi}_{P E}\left(\overline{\mathcal{J}}_{P E}^{*}+\sigma^{2}\right)$. Here, $\overline{\mathcal{J}}_{P E}^{*}$ is obtained from solving (11) and plugged. Then, the Lagrangian can be formed as

$$
\begin{gathered}
\mathcal{L}=\alpha^{2} I_{D C}^{2} \rho^{2} \mathbf{b}^{T} \mathbf{H}_{U E}^{\Theta} \mathbf{b}-\lambda\left(\alpha^{2} I_{D C}^{2} \rho^{2} \mathbf{b}^{T} \overline{\mathbf{H}}_{P E}^{\Theta} \mathbf{b}-C\right) \\
+\mu_{-}^{T}(\Theta \mathbf{b}+\mathbb{1})-\mu_{+}^{T}(\Theta \mathbf{b}-\mathbb{1})
\end{gathered}
$$

where $\lambda \in \mathbb{R}$ and $\mu_{-}, \mu_{+} \in \mathbb{R}^{N}$ denote the Lagrange multipliers. In order to let (17) yield an analytic, non-trivial minimum with respect to $\mathbf{b}$, we assume the second constraint of (16b) is slack, that is, all the elements of $\Theta \mathbf{b}$ are in between -1 and 1 (exclusive). Then, this assumption is equivalent to

$$
\mu_{-}=\mu_{+}=\mathbb{D}
$$

according to the local sensitivity analysis in [36], where $\mathbb{0}$ denotes the all-zeros column vector. The condition stated in (18) can be satisfied depending upon $C$. For a small $C$, any element of $\Theta \mathbf{b}$ cannot reach \pm 1 ; in other words, the second constraint in (16b) is inactive.

It is worthwhile to mention that the channel strength in VLC networks mainly depends on the distance between the transmitter and receiver. Thus, the UE is likely to receive the data signal principally from the closest LED transmitter. In contrast, the other far-off transmitters utilize less power to transmit data signals or are silent under PLS transmission schemes designed for VLC systems [25]. In other words, the signals transmitted from the LEDs located far from the UE would not considerably contribute to the information signal power received at the UE, while it would allow more opportunities for the PEDs that might exist in the entire room to overhear the information signals. In this sense, it would be wise to set $\bar{\xi}_{P E}$ such that the nearest LED to the UE broadcasts the data signal with a high transmit power by setting its beamforming weight close to \pm 1 ; in contrast, the other faroff LEDs' beamforming weights need to be set close to 0 . Therefore, when designing a PLS beamforming method in the VLC system, it is likely that the condition (18) is satisfied.

With assuming that (18) holds, calculating the partial derivative of $\mathcal{L}$ with respect to $\mathbf{b}$ and setting it equal to zero yield

$$
\mathbf{H}_{U E}^{\Theta} \mathbf{b}=\lambda \overline{\mathbf{H}}_{P E}^{\Theta} \mathbf{b}
$$


where the rank of $\overline{\mathbf{H}}_{P E}^{\Theta}$ is less than or equal to $N-N_{A}$; that is, $\overline{\mathbf{H}}_{P E}^{\Theta}$ is singular. Thus, we utilize the inverse free preconditioned Krylov subspace projection method, which is an efficient algorithm for obtaining a few extreme eigenpairs of the symmetric generalized eigenvalue problem [37]. In this method, an eigenvector estimate $\mathbf{b}_{k}$ at the $k$ th iteration continuously improves by the Rayleigh-Ritz projection onto the Krylov subspace

$$
\begin{aligned}
& K_{n}\left(\mathbf{H}_{U E}^{\Theta}-\zeta_{k} \overline{\mathbf{H}}_{P E}^{\Theta}, \mathbf{b}_{k}\right) \\
& =\operatorname{span}\left(\mathbf{b}_{k},\left(\mathbf{H}_{U E}^{\Theta}-\zeta_{k} \overline{\mathbf{H}}_{P E}^{\Theta}\right) \mathbf{b}_{k}, \ldots,\left(\mathbf{H}_{U E}^{\Theta}-\zeta_{k} \overline{\mathbf{H}}_{P E}^{\Theta}\right)^{n} \mathbf{b}_{k}\right)
\end{aligned}
$$

where $\zeta_{k}=\mathbf{b}_{k}^{T} \mathbf{H}_{U E}^{\Theta} \mathbf{b}_{k} / \mathbf{b}_{k}^{T} \overline{\mathbf{H}}_{P E}^{\Theta} \mathbf{b}_{k}$ signifies the Rayleigh quotient, and $n$ denotes a parameter to be selected, and $\operatorname{span}\left(\mathbf{v}_{1}, \mathbf{v}_{2}, \ldots, \mathbf{v}_{N}\right)$ represents the set of all linear combinations of the vectors. We use a MATLAB program eigifp [38] to conduct the entire procedure explained above, which takes two inputs, $\mathbf{H}_{U E}^{\Theta}$ and $\overline{\mathbf{H}}_{P E}^{\Theta}$, and returns the estimated maximum eigenvalue and corresponding eigenvector.

Then, we have

$$
\mathcal{D}_{U E}=\alpha^{2} I_{D C}^{2} \rho^{2} \mathbf{b}^{T} \mathbf{H}_{U E}^{\Theta} \mathbf{b}=\alpha^{2} I_{D C}^{2} \rho^{2} \lambda \mathbf{b}^{T} \overline{\mathbf{H}}_{P E}^{\Theta} \mathbf{b} \leq \lambda_{\max } C .
$$

Here, it is noted that the maximum of the received data power at the UE $\mathcal{D}_{U E}$ is proportional to the maximum eigenvalue $\lambda_{\max }$. Thus, the optimal vector $\mathbf{b}^{*}$ can be evaluated by scaling the eigenvector $\mathbf{b}_{\max }$ corresponding to $\lambda_{\max }$ such that $\alpha^{2} I_{D C}^{2} \rho^{2} \mathbf{b}^{* T} \overline{\mathbf{H}}_{P E}^{\Theta} \mathbf{b}^{*}=C$. Then, the optimal beamforming vector $\mathbf{w}^{*}$ can be calculated as $\mathbf{w}^{*}=\Theta \mathbf{b}^{*}$. Finally, we must check the feasibility of $\mathbf{w}^{*}$ by confirming the satisfaction of (18). If all the elements of $\mathbf{w}^{*}$ are in between -1 and 1 (exclusive), (18) is satisfied; hence, the corresponding solution $\mathbf{w}^{*}$ is the optimal solution.

On the contrary, if any element of the obtained $\mathbf{w}^{*}$ is not in between -1 and 1 , the second constraint in (16b) is not slack; therefore, (18) cannot be achieved. In this case, the suboptimal vector $\mathbf{b}^{*}$ can be numerically obtained by using the $\mathrm{CCP}$ in a similar manner to Algorithm 1, where the objective function in (16a) needs to be converted to

$$
\alpha^{2} I_{D C}^{2} \rho^{2} \mathbf{b}_{k}^{T} \mathbf{H}_{U E}^{\Theta} \mathbf{b}_{k}+2 \alpha^{2} I_{D C}^{2} \rho^{2}\left(\mathbf{H}_{U E}^{\Theta} \mathbf{b}_{k}\right)^{T}\left(\mathbf{b}-\mathbf{b}_{k}\right) .
$$

\section{Step 3: Find $\rho^{\star}$}

To find the optimal power splitter coefficient $\rho^{\star}$ that yields the maximum SINR of the UE $\gamma_{U E}^{\star}$, we adopt the GSS method [39]. The GSS is a technique for finding a minimum or maximum of a function inside a specified interval. Here, it can be expected that $\gamma_{U E}$ is a quasiconcave function of $\rho$ over the interval $[0,1]$. When $\rho=0, \gamma_{U E}=0$. In contrast, when $\rho=1$, i.e., without jamming interference, the LED transmitters should decrease their information transmit power to satisfy the constraint regarding the PEDs, which results in a decrease of $\gamma_{U E}$. Thus, it is expected that there would be one optimal point of $\rho$ in the middle of $[0,1]$ that yields $\gamma_{U E}^{\star}$. The details for finding the optimal power splitter coefficient $\rho^{\star}$ are given in Algorithm 2.

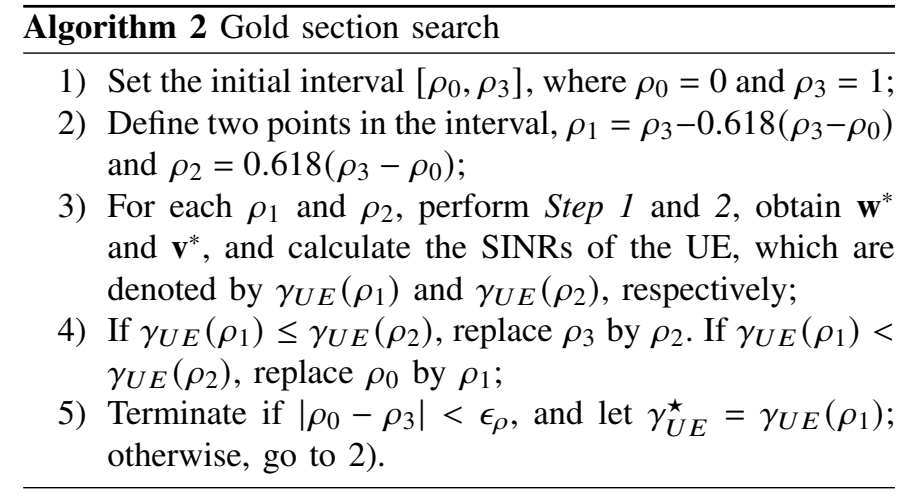

TABLE I: Parameters Settings

\begin{tabular}{c|c}
\hline \multicolumn{2}{c}{ Room configuration } \\
\hline Height from the work plane $(\mathrm{H})$ & $2.2 \mathrm{~m}$ \\
Length $(\mathrm{L}) \times$ Width $(\mathrm{W})$ & $20 \times 20 \mathrm{~m}^{2}$ \\
Number of LEDs per fixture & 16 \\
\hline \multicolumn{2}{c}{ LED characteristics } \\
\hline Average optical power per LED & $0.5 \mathrm{~W}$ \\
Current-to-light conversion efficiency $\eta$ & $5 \mathrm{~W} / \mathrm{A}$ \\
Nominal half-intensity angle $\phi_{1 / 2}$ & $60^{\circ}$ \\
Modulation index $\alpha$ & 0.4 \\
\hline Receiver characteristics \\
\hline Photodetector's physical area $A_{\mathrm{PD}, k}$ & $1 \mathrm{~cm}{ }^{2}$ \\
Photodetector's responsivity $R_{k}$ & $0.54 \mathrm{~mA} / \mathrm{mW}$ \\
Noise power $\sigma^{2}$ & $-98.33 \mathrm{dBm}$ \\
Field of View $\Psi_{c}$ & $90^{\circ}$ \\
Transimpedance amplifier gain $T_{k}$ & $50 \mathrm{~V} / \mathrm{A}$ \\
\hline
\end{tabular}

Now, we have $\rho^{\star}$ and its corresponding beamforming and jamming vectors $\mathbf{w}^{*}$ and $\mathbf{v}^{*}$, which yield the maximum SINR of the UE $\gamma_{U E}^{\star}$. Finally, we set the final solution of the original problem in (7), i.e., $\left\{\rho^{\star}, \mathbf{w}^{\star}, \mathbf{v}^{\star}\right\}$, to $\left\{\rho^{\star}, \mathbf{w}^{*}, \mathbf{v}^{*}\right\}$. The final solution of (7) can be found in tens of seconds by using MATLAB on a standard PC (Intel i7, 3.4 GHz). It is worth noting that the computational complexity of the proposed scheme may seem too high to be realized in a practical VLC system. Thus, additional efforts to reduce the complexity would be required, which may include quantizing the beamforming and jamming vectors and employing codebook design as in [40], [41]. These efforts would be an appealing topic for future work.

\section{NUMERICAL RESULTS}

In this section, the numerical results are provided to verify the performance of the proposed cooperative beamforming and jamming method. Table I provides the room configuration and simulation parameters. The $10 \times 10$ LED transmitters are uniformly distributed on a square lattice in a $20 \times 20 \mathrm{~m}^{2}$ room and their locations are provided by $\left(x_{T}, y_{T}, 2.2\right)$, where $x_{T}, y_{T} \in\{ \pm 1, \pm 3, \pm 5, \pm 7, \pm 9\}$. Besides, the emitted light is assumed to be "warm-white" with luminous efficiency 284 $\mathrm{lm} / \mathrm{W}$ [42, Table 3.2]. According to the Lumen method [43], the considered LED arrangement yields the light level of 410 lx in the room, which satisfies the lighting standards, e.g., from 400 to $10001 x$ for a typical office [44]. 


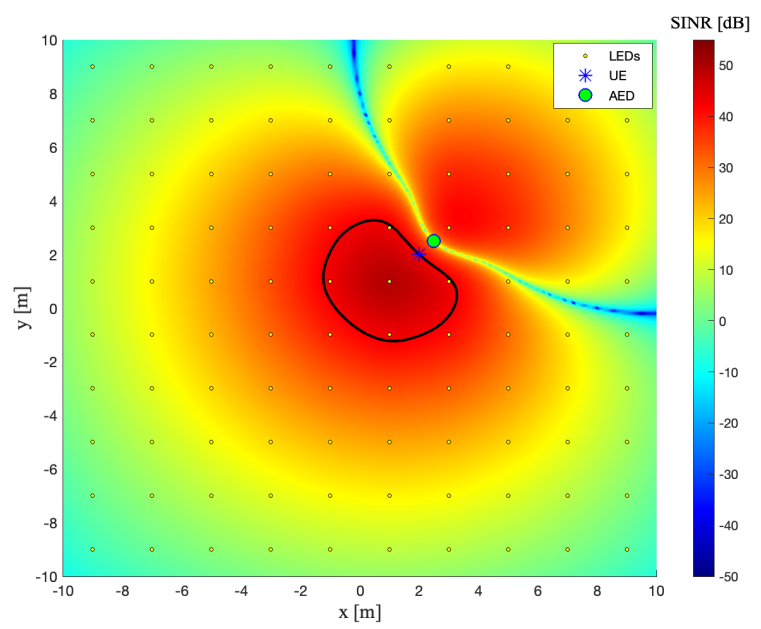

(a) The traditional ZF beamforming in [8]

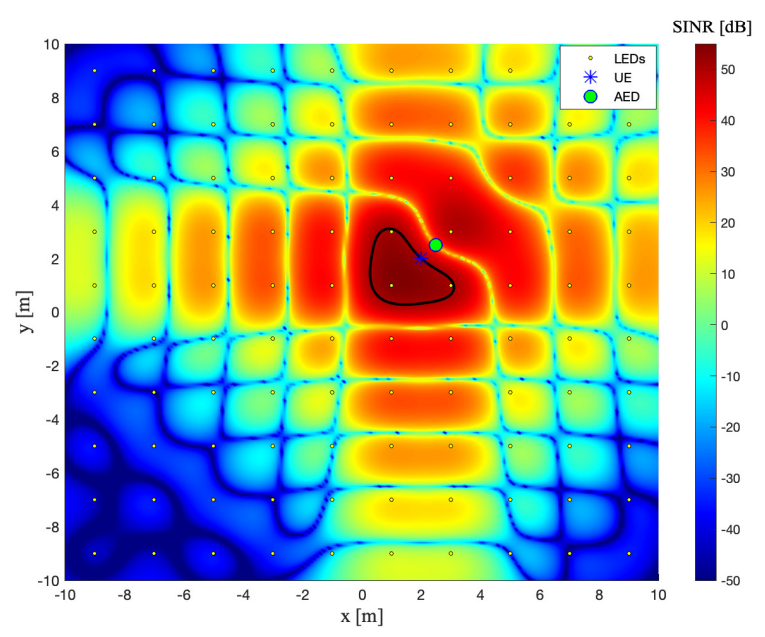

(c) The enhanced ZF beamforming in [24]

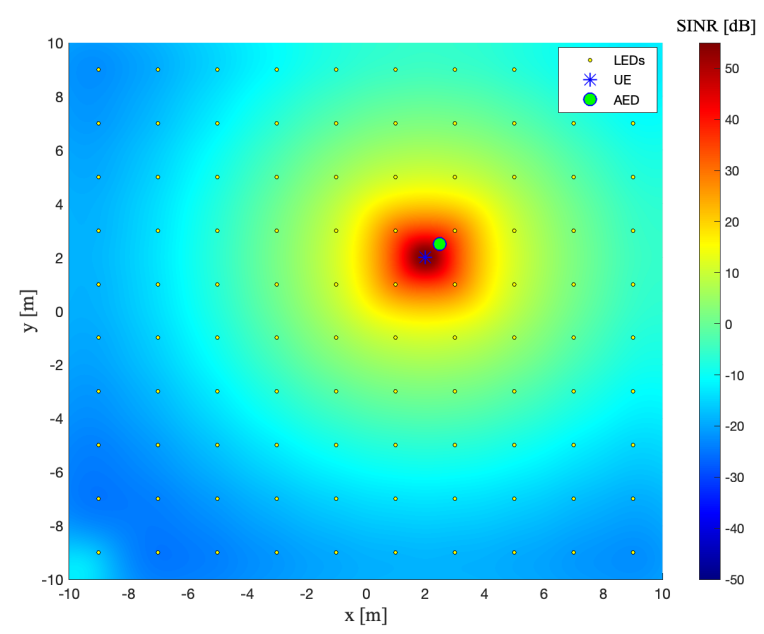

(b) The traditional artificial noise transmission in [8]

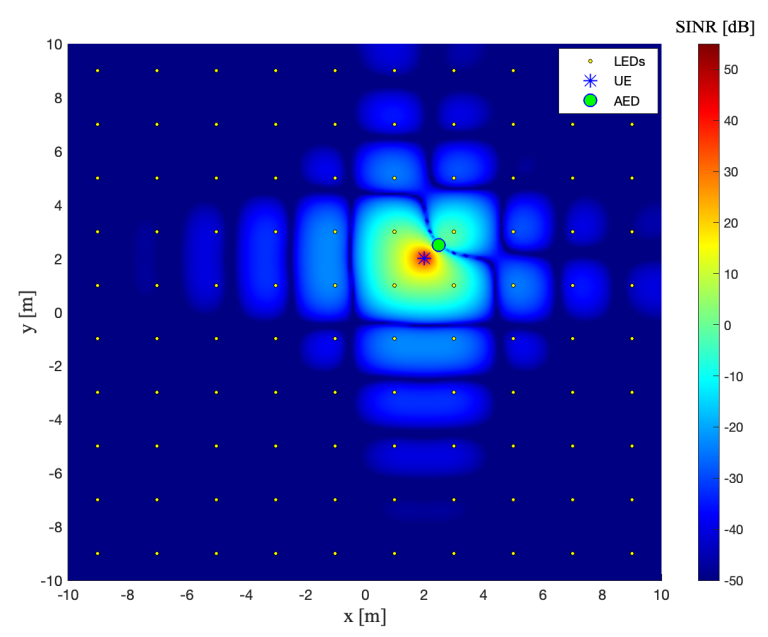

(d) The proposed cooperative beamforming and jamming scheme in (7)

Fig. 2: The SINR based on the receiver locations for different transmission schemes, where the UE and the AED are placed at $(2,2) \mathrm{m}$ and $(2.5,2.5) \mathrm{m}$, respectively. $\bar{\xi}_{P E}=10^{-3}$ is used for (d).

Figs. 2(a), (b), (c), and (d) show the SINR values according to the receiver locations when the traditional $\mathrm{ZF}$ beamforming [8], the traditional artificial noise transmission [8], the enhanced ZF beamforming for passive and active EDs [24], and the proposed cooperative beamforming and jamming scheme are adopted, respectively. The first three results are provided as benchmarks. For all the cases, the UE and the AED are placed at $(2,2) \mathrm{m}$ and $(2.5,2.5) \mathrm{m}$, respectively. Also, the black contours indicate the areas in which the SINR is higher than that of the UE, i.e., $\mathcal{A}$ in (5), in which the PEDs can retain a higher SINR than the UE and cause a secrecy outage. To give a clear presentation, the figures show the SNR down to $-50 \mathrm{~dB}$, although the AED's SINRs in Figs. 2(a), (c), and (d) are less than this value. For the traditional ZF scheme [8], it utilizes the beamforming vector as

$$
\mathbf{w}_{\mathrm{ZF}}=k_{\mathrm{ZF}} \Psi_{A E} \Psi_{A E}^{T} \mathbf{h}_{U E}
$$

where $k_{\mathrm{ZF}}$ is a constant such that $\left|\mathbf{w}_{\mathrm{ZF}}\right| \leq \mathbb{1}$. For the traditional artificial noise transmission [8], the beamforming and jamming vectors are obtained as

$$
\mathbf{w}_{\mathrm{AN}}=k_{\mathrm{AN}} \alpha I_{D C} \rho \hat{\mathbf{h}}_{U E}
$$

and

$$
\mathbf{v}_{\mathrm{AN}}=k_{\mathrm{AN}} \alpha I_{D C} \frac{1-\rho}{N-1} \sum_{i=1}^{N-1} \hat{\varphi}_{\mathrm{B}_{i}}
$$

respectively, where $\hat{\mathbf{h}}_{U E}=\mathbf{h}_{U E} /\left\|\mathbf{h}_{U E}\right\|_{1}$, and $\hat{\varphi}_{\mathrm{B}_{i}} \in \mathbb{R}^{N}$ for $i \in\{1,2, \ldots, N-1\}$ constitute a basis for the nullspace of $\mathbf{h}_{U E}^{T}$ and are normalized such that $\left\|\hat{\varphi}_{\mathrm{B}_{i}}\right\|_{1}=1, \forall i \in\{1,2, \ldots, N-1\}$. $\|\cdot\|_{1}$ denotes the 1 -norm operator. $k_{\mathrm{AN}}$ is a constant such that the peak constraint

$$
k_{\mathrm{AN}}\left(\rho\left|\hat{\mathbf{h}}_{B}\right|+\frac{1-\rho}{N-1} \sum_{i=1}^{N-1}\left|\hat{\varphi}_{B_{i}}\right|\right) \leq \mathbb{1}
$$

is satisfied. Also, for the enhanced $\mathrm{ZF}$ beamforming for active and passive EDs [24], $\bar{\rho}_{P E}=36.6 \mathrm{~dB}$ is used, where $\bar{\rho}_{P E}$ 
represents the target constraint on the average SNR of a PED. Notice that the enhanced ZF beamforming scheme also takes both AEDs and PEDs into account, but it does not employ jamming signals.

Fig. 2(a) shows that the traditional ZF beamforming method appears to efficiently suppress the SINR of the AEDs to zero by steering its beam onto the null space of the AED channel. However, this scheme aims only at forcing the SINR of AED to zero. It does not maximize the UE's SINR, nor does it constrain the PEDs' average SINR. As a result, it yields a spacious contoured area where the SINR is higher than that of the UE, which would result in a high SOP. In Fig. 2(b), it is shown that the artificial noise transmission can somewhat constrain the average SINR of the PEDs; however, the AED can retain a high SINR since this scheme does not take the AED into account. Also, this artificial interference scheme merely tries to emit jamming signals toward the nullspace of the UE's channel with the hope that interference is caused at the ED sites. In other words, this method leaves the resulting secrecy performance depending on the nullspace of the UE; thereby, this method cannot always guarantee to maximize the interference at the PED sites. In Fig. 2(c), we can note that the enhanced ZF beamforming scheme effectively copes with both the AED and PEDs by minimizing the AED's SINR and maximizing the UE's SINR, as well as constraining the average SINR of PEDs. However, it still yields a spacious contoured area in which the SINR is higher than that of the UE to cope with the AED located close to the UE.

In contrast, Fig. 2(d) shows that the proposed cooperative beamforming and jamming strategy effectively constrains the SINRs of both the AED and PEDs, while maximizing the SINR of the UE. The UE can retain the highest SINR (42.3 $\mathrm{dB}$ ) in the room, while the AED's SINR is suppressed to zero and the PEDs' average SINR $(5.00 \mathrm{~dB})$ is considerably constrained. Looking at Table II showing the SINRs of the UE, AED, and PEDs, we can confirm that the proposed scheme can secure VLC transmissions against the AED and PEDs simultaneously, unlike the traditional ZF beamforming and artificial noise schemes that deal with either AED or PEDs, but not both. Note that although the UE's SINRs with the benchmarks appear higher than that of the proposed scheme, the SINR gap between the UE and the PEDs is much larger with the proposed scheme $(37.3 \mathrm{~dB})$ compared to the three benchmarks $(9.1 \mathrm{~dB}, 27.6 \mathrm{~dB}, 13.9 \mathrm{~dB})$.

On the other hand, the fact that the enhanced ZF beamforming scheme in Fig. 2(c) can effectively cope with the AED and PEDs simultaneously might evoke an impression that the enhanced $\mathrm{ZF}$ beamforming is already sufficient to provide a secured transmission to the UE unless the PEDs are present close to the UE, i.e., in the contoured area. However, in practice, the potential eavesdropping scenario can be the ED with powerful receiver front-end specifications more superior than those of the UE, such as a bigger physical PD area, a higher PD responsivity, a higher refractive index, etc. Thus, in Fig. 3, we evaluate the SINR according to the receiver locations when the PD's size is enlarged from $1 \mathrm{~cm}^{2}$ to $10 \mathrm{~cm}^{2}$ to see how the augmentation of the PEDs' receiver

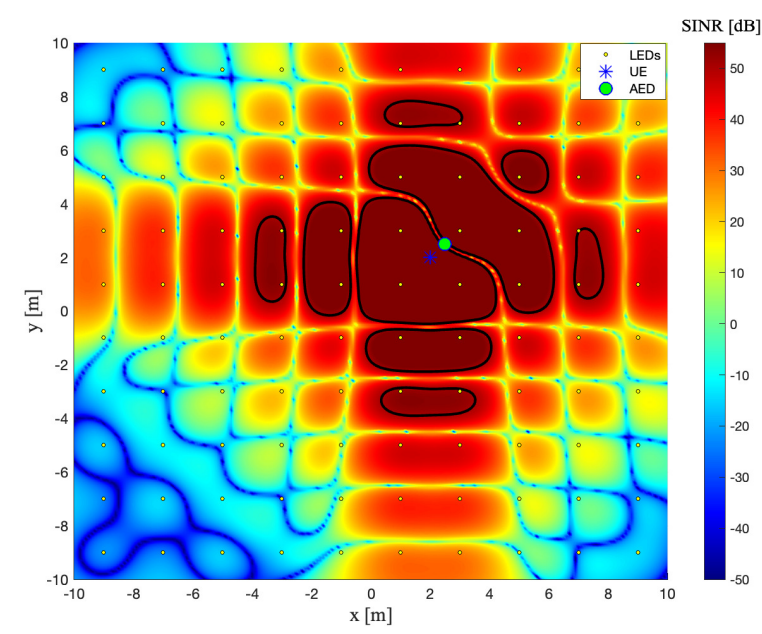

(a) The enhanced ZF beamforming in [24]

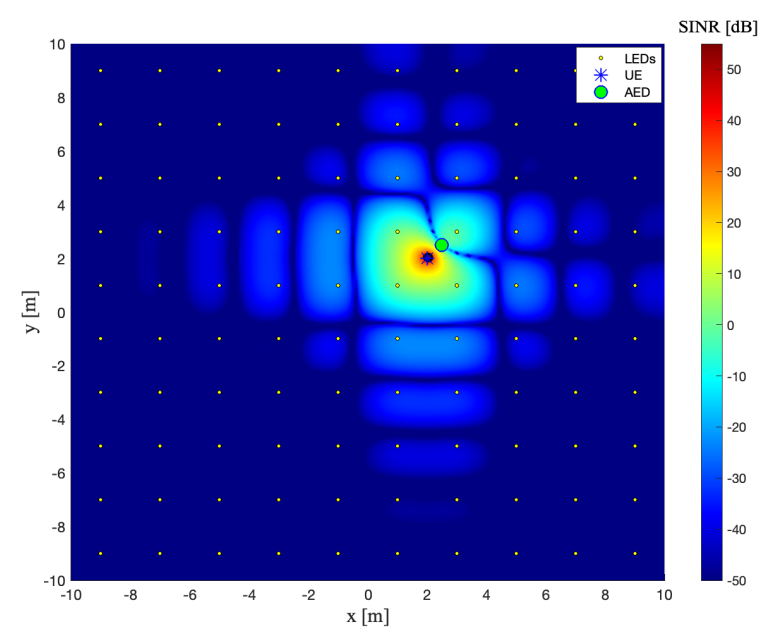

(b) The proposed cooperative beamforming and jamming scheme in (7)

Fig. 3: The SINR according to the receiver locations for different transmission schemes when the PD's physical area of the PEDs $A_{\mathrm{PD}, P E_{q}}$ is increased from $1 \mathrm{~cm}^{2}$ to $10 \mathrm{~cm}^{2}$. The UE and the AED are placed at $(2,2) \mathrm{m}$ and $(2.5,2.5) \mathrm{m}$, respectively.

would affect the secrecy performance ${ }^{4}$. Note that all the other system and environment parameters are identical to those of Figs. 2(c) and (d). Fig. 3(a) verifies that the PEDs under the enhanced ZF beamforming scheme can significantly increase their SINRs by $20 \mathrm{~dB}$ over the whole range; even worse, the contoured area in which the SINR is higher than the UE is dramatically increased from $5.2 \mathrm{~m}^{2}$ to $56.7 \mathrm{~m}^{2}$, compared to Fig. 2(c). In other words, the PEDs with the larger PD size can more easily retain a higher SINR than the UE and cause the secrecy outage. In contrast, under the proposed cooperative beamforming and jamming scheme in Fig. 3(b), it is shown that the SINR remains almost the same over the whole room area, and the UE still retains the highest SINR. This result

${ }^{4}$ Considering the channel model in (2), other better specifications of the ED can be considered in the same way. 
TABLE II: SINRs in Fig. 2

\begin{tabular}{c|c|c|c|c}
\hline & Zero-Forcing [8] & $\begin{array}{l}\text { Artificial } \\
\text { Noise [8] }\end{array}$ & $\begin{array}{l}\text { Enhanced } \\
\text { Zero-Forcing in [24] }\end{array}$ & $\begin{array}{l}\text { Proposed } \\
\text { scheme (7) }\end{array}$ \\
\hline$\gamma_{U E}(\mathrm{~dB})$ & 45.7 & 54.6 & 50.5 & 42.3 \\
\hline$\gamma_{A E_{1}}(\mathrm{~dB})$ & -112 & 44.2 & -98 & -142 \\
\hline $\mathbb{E}\left[\gamma_{P E_{q}}\right](\mathrm{dB})$ & 36.6 & 27.0 & 36.6 & 5.00 \\
\hline$|\mathcal{A}|\left(\mathrm{m}^{2}\right)$ & 13.9 & 0 & 5.2 & 0 \\
\hline
\end{tabular}

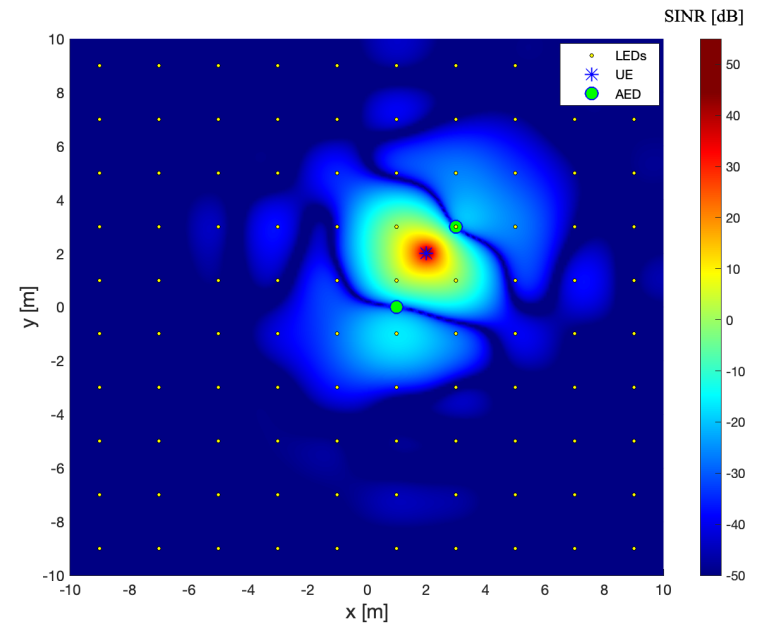

Fig. 4: The SINR based on the receiver locations when multiple AEDs are present. The UE and two AEDs are placed at $(2,2) \mathrm{m},(3,3) \mathrm{m}$ and $(0,1) \mathrm{m}$, respectively. $\bar{\xi}_{P E}=10^{-3}$ is used.

verifies that the PEDs under the proposed cooperative scheme cannot improve their SINR by raising the PD area since the PEDs receive more of the jamming and information signals by the larger PD.

Fig. 4 shows the SINRs according to the receiver locations in the presence of two AEDs. The UE is located at $(2,2)$, and the AEDs are located at $(3,3)$ and $(0,1)$, respectively. Even for multiple AEDs, the proposed cooperative beamforming and jamming scheme is shown to suppress the SINRs of the two AEDs to zero by laying the beamforming vectors into the null spaces of AEDs' channel matrix. Moreover, the proposed scheme lays the jamming intensity vector onto the null space of the UE's channel vector, thereby, it could maximize the interference at PEDs site without causing it at the UE's site.

Figs. 5(a) and (b) show the SINR gaps between the UE and the AED and between the UE and the PEDs, respectively, according to the different radii of the circle where one AED is randomly located. The circle is centered at the UE located at $(2,2)$. Here, a smaller radius implies that the AED is likely closer to the UE. The results are obtained by MonteCarlo simulation with $10^{3}$ trials, where we generate a random location of the AED in the circle and obtain the beamforming and jamming vectors for each transmission scheme given the locations of the UE and the AED. Then we evaluate the SINRs of the UE and EDs. In Fig. 5(a), the SINR gap between the UE and the AED with the proposed scheme, i.e., $\gamma_{U E}[\mathrm{~dB}]-\gamma_{A E_{1}}[\mathrm{~dB}]$, appears the highest over the entire region of radii, compared to the other benchmark schemes. This superiority implies that the proposed scheme can effectively secure the information transmission against the AED regardless of the AED's location. On the other hand, when the AED is located very closer to the UE, the SINR gap with the traditional artificial noise scheme is minimal (e.g., $2.01 \mathrm{~dB}$ for $0.5 \mathrm{~m}$ ), which implies that the traditional artificial noise scheme cannot adequately prevent the AED from wiretapping.

In addition, in Fig. 5(b), the gap between the UE's SINR and the PEDs' average SINR with the proposed scheme, i.e., $\gamma_{U E}[\mathrm{~dB}]-\mathbb{E}\left[\gamma_{P E_{q}}\right][\mathrm{dB}]$, also appears the highest over the entire region of radii, compared to the other benchmark schemes. Notably, the proposed scheme's secrecy performance in restricting the PEDs is more superior than even the artificial noise scheme that aims at curbing the PEDs solely. Besides, when the AED is getting closer to the UE, the gaps between the UE's SINR and the PEDs' average SINR under the enhanced and traditional ZF schemes decrease from $16.9 \mathrm{~dB}$ and $13.7 \mathrm{~dB}$ for $4 \mathrm{~m}$ to $9.11 \mathrm{~dB}$ and $3.98 \mathrm{~dB}$ for $0.5 \mathrm{~m}$, respectively. This observation implies that the transmission schemes without employing artificial noise cannot rigorously constrain PEDs in the presence of the AEDs close to the UE; thus, more eavesdropping opportunities are leaked to the PEDs.

Fig. 5(c) shows the SOP for the different schemes according to the different circle radii. The figure shows that the proposed scheme and the traditional artificial noise scheme yield zero SOPs, i.e., the UE retains the highest SINR in the room with those schemes. In contrast, the SOPs with the enhanced and traditional ZF schemes appear very high when the AED is close to the UE, signifying that the enhanced and traditional ZF might not suitably secure the VLC systems in terms of the SOP metric depending on the AED's site.

\section{CONCLUSION}

This paper developed a novel cooperative beamforming and jamming method to prevent both the AEDs and hidden PEDs from eavesdropping on the VLC transmission. Compared to the traditional VLC jamming strategies that could deal with either passive or active EDs, the proposed cooperative strategy could enhance secrecy performance against both the AEDs and PEDs. We formulated an optimization problem for finding beamforming and jamming vectors and a power splitter coefficient. The objective was to maximize the SINR of the UE while fully suppressing the AEDs' SINRs and restricting the PEDs' average SINR. The suboptimal beamforming and jamming vectors can be analytically and numerically found 


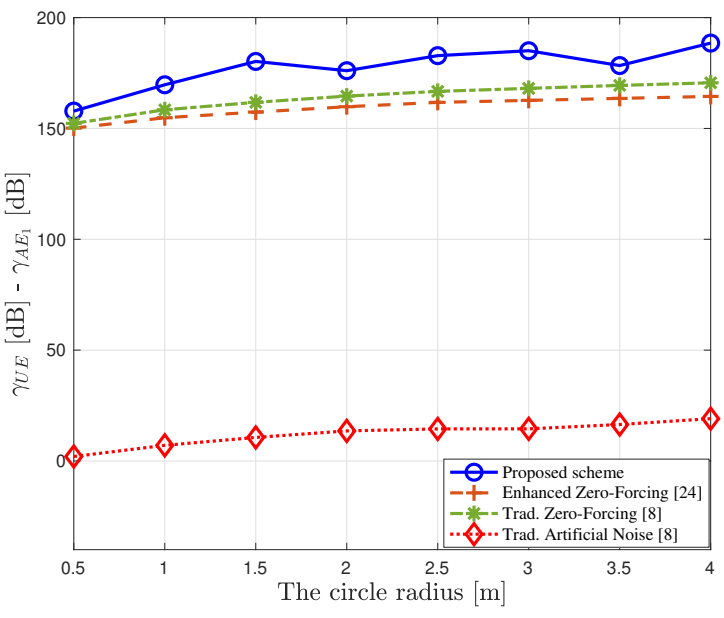

(a) The SINR gap between the UE and the AED

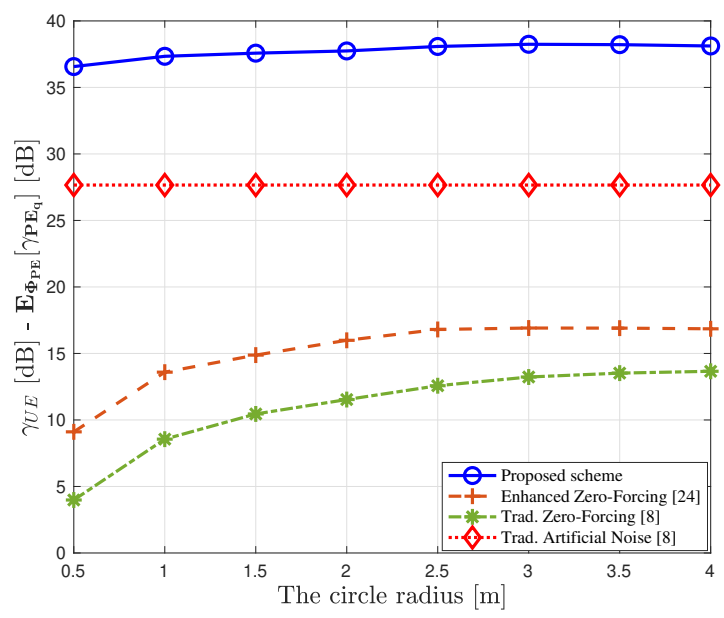

(b) The SINR gap between the UE and the PEDs

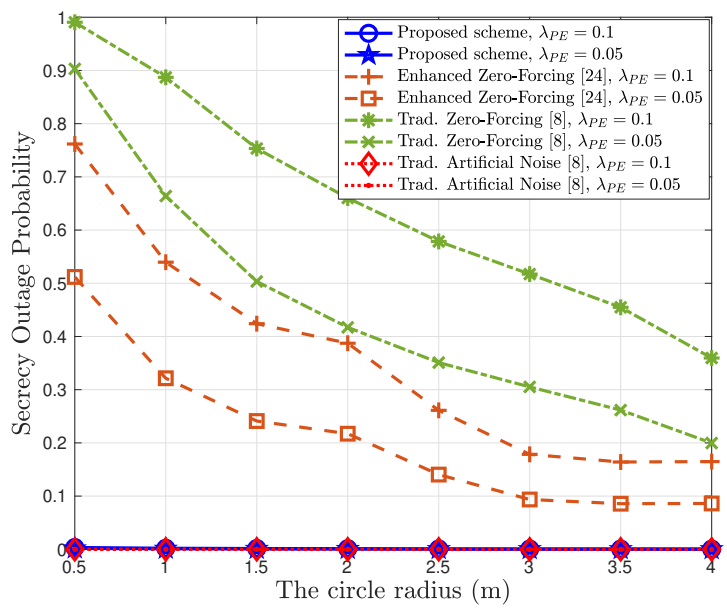

(c) The secrecy outage probability

Fig. 5: The SINR gap and the SOP are shown for different radii of the circle where one AED is randomly located. The UE is located at $(2,2)$, and the circle's center is also at the UE site. $\bar{\xi}_{P E}=10^{-3}$ is used. via an inverse free preconditioned Krylov subspace projection method and the CCP. The optimal power splitter coefficient can be numerically obtained via the golden section search method. The numerical results verified that the proposed cooperative beamforming and jamming scheme could provide a secured transmission for the VLC systems in which active and passive EDs exist and yield better secrecy performance relative to three benchmarks: the traditional ZF beamforming, the scheme that injects artificial noise into the system, and the enhanced ZF beamforming.

\section{REFERENCES}

[1] H. Haas, L. Yin, Y. Wang, and C. Chen, "What is LiFi?" J. Lightw. Technol., vol. 34, no. 6, pp. 1533-1544, Mar. 2016.

[2] M. M. Mowla, I. Ahmad, D. Habibi, and Q. V. Phung, "A green communication model for 5G systems," IEEE Trans. Green Commun. Netw., vol. 1, no. 3, pp. 264-280, 2017.

[3] E. Tews and M. Beck, "Practical attacks against WEP and WPA," in ACM WiSec in Zurich, Switzerland, Mar. 2009, pp. 79-86.

[4] M. Bloch and J. Barros, Physical-Layer Security: From Information Theory to Security Engineering, 1st ed. New York, NY, USA: Cambridge University Press, 2011.

[5] A. Mostafa and L. Lampe, "Physical-layer security for MISO visible light communication channels," IEEE J. Sel. Areas Commun., vol. 33, no. 9, pp. 1806-1818, Sept. 2015.

[6] —- "Securing visible light communications via friendly jamming," in IEEE Globecom Workshops in Austin, USA, Dec. 2014, pp. 524-529.

[7] —, "Pattern synthesis of massive LED arrays for secure visible light communication links," in IEEE ICCW in London, U.K., June 2015, pp. $1350-1355$.

[8] - "Physical-layer security for indoor visible light communications," in IEEE ICC in Sydney, Australia, June 2014, pp. 3342-3347.

[9] — "Optimal and robust beamforming for secure transmission in MISO visible-light communication links," IEEE Trans. Signal Process., vol. 64, no. 24, pp. 6501-6516, Dec. 2016.

[10] H. Zaid, Z. Rezki, A. Chaaban, and M. S. Alouini, "Improved achievable secrecy rate of visible light communication with cooperative jamming," in IEEE GlobalSIP in Orlando, U.S.A., Dec. 2015, pp. 1165-1169.

[11] M. A. Arfaoui, Z. Rezki, A. Ghrayeb, and M. S. Alouini, "On the secrecy capacity of MISO visible light communication channels," in IEEE Globecom in Washington D.C., USA, Dec. 2016, pp. 1-7.

[12] J. Wang, H. Ge, M. Lin, J. Wang, J. Dai, and M. Alouini, "On the secrecy rate of spatial modulation-based indoor visible light communications," IEEE J. Sel. Areas Commun., vol. 37, no. 9, pp. 2087-2101, 2019.

[13] T. V. Pham and A. T. Pham, "On the secrecy sum-rate of MU-VLC broadcast systems with confidential messages," in 2016 CSNDSP in Prague, Czech Republic, July 2016, pp. 1-6.

[14] T. V. Pham, T. Hayashi, and A. T. Pham, "Artificial-noise-aided precoding design for multi-user visible light communication channels," IEEE Access, vol. 7, pp. 3767-3777, 2019.

[15] T. V. Pham and A. T. Pham, "Energy efficient artificial noise-aided precoding designs for secured visible light communication systems," IEEE Transactions on Wireless Communications, vol. 20, no. 1, pp. 653666, 2021.

[16] J. Chen and T. Shu, "Statistical modeling and analysis on the confidentiality of indoor vlc systems," IEEE Transactions on Wireless Communications, vol. 19, no. 7, pp. 4744-4757, 2020.

[17] J.-Y. Wang, Y. Qiu, S.-H. Lin, J.-B. Wang, Q. Wang, and B. Zhang, "Performance analysis and improvement for secure vlc with slipt and random terminals," IEEE Access, vol. 8, pp. 73645-73 658, 2020.

[18] M. A. Arfaoui, M. D. Soltani, I. Tavakkolnia, A. Ghrayeb, M. Safari, C. M. Assi, and H. Haas, "Physical layer security for visible light communication systems: A survey," IEEE Commun. Surveys Tuts., vol. 22, no. 3, pp. 1887-1908, 2020.

[19] M. Obeed, A. M. Salhab, M. Alouini, and S. A. Zummo, "On optimizing VLC networks for downlink multi-user transmission: A survey," CoRR, vol. abs/1808.05089, 2018. [Online]. Available: http://arxiv.org/abs/1808.05089

[20] T. Komine and M. Nakagawa, "Fundamental analysis for visible-light communication system using LED lights," IEEE Trans. Consum. Electron, vol. 50, no. 1, pp. 100-107, Feb. 2004. 
[21] I. Marin-Garcia, V. Guerra, and R. Perez-Jimenez, "Study and Validation of Eavesdropping Scenarios over a Visible Light Communication Channel," Sensors, vol. 17, no. 12, p. 2687, Nov. 2017.

[22] S. Cho, G. Chen, and J. P. Coon, "Enhancement of physical layer security with simultaneous beamforming and jamming for visible light communication systems," IEEE Trans. Inf. Forens. Security, vol. 14, no. 10 , pp. 2633-2648, Oct. 2019.

[23] —_ "Securing visible light communications with spatial jamming," in IEEE ICC in Shanghai, China, May 2019, pp. 1-6.

[24] _ " "Zero-forcing beamforming for active and passive eavesdropper mitigation in visible light communication systems," IEEE Trans. Inf. Forens. Security, vol. 16, pp. 1495-1505, 2021.

[25] _ _ "Securing visible light communication systems by beamforming in the presence of randomly distributed eavesdroppers," IEEE Trans. Wireless Commun., vol. 17, no. 5, pp. 2918-2931, May 2018.

[26] A. Baddeley, E. Rubak, and R. Turner, Spatial point patterns: methodology and applications with R. CRC Press, 2015.

[27] G. Geraci, S. Singh, J. G. Andrews, J. Yuan, and I. B. Collings, "Secrecy rates in broadcast channels with confidential messages and external eavesdroppers," IEEE Trans. Wireless Commun., vol. 13, no. 5, pp. 2931-2943, May 2014.

[28] H. Dogan, O. Şayll, and E. Panayirci, "Pilot assisted channel estimation for asymmetrically clipped optical OFDM over visible light channels," in 2016 IEEE BlackSeaCom in Varna, Bulgaria, 2016, pp. 1-4.

[29] B. Aly, "Performance analysis of adaptive channel estimation for U-OFDM indoor visible light communication," in 2016 NRSC in Aswan, Egypt, 2016, pp. 217-222.

[30] S. Cho, G. Chen, H. Chun, J. P. Coon, and D. O'Brien, "Impact of multipath reflections on secrecy in VLC systems with randomly located eavesdroppers," in IEEE WCNC in Barcelona, Spain, Apr. 2018, pp. $1-6$.

[31] Z. Yi and I. Kim, "Joint optimization of relay-precoders and decoders with partial channel side information in cooperative networks," IEEE J. Sel. Areas Commun., vol. 25, no. 2, pp. 447-458, 2007.

[32] H. Konno, "Maximizing a convex quadratic function over a hypercube," Journal of the Operations Research Society of Japan, vol. 23, no. 2, pp. $171-189,1980$.

[33] T. Lipp and S. Boyd, "Variations and extension of the convex-concave procedure," Optimization and Engineering, vol. 17, no. 2, pp. 263-287, June 2016.

[34] P. T. Boggs and J. W. Tolle, "Sequential quadratic programming," Acta Numerica, vol. 4, pp. 1-51, 1995.

[35] I. I. Dikin, "Iterative solution of problems of linear and quadratic programming," Sov. Math., Dokl., vol. 8, pp. 674-675, 1967.

[36] S. Boyd and L. Vandenberghe, Convex Optimization. New York, NY, USA: Cambridge University Press, 2004.

[37] G. H. Golub and Q. Ye, "An inverse free preconditioned Krylov subspace method for symmetric generalized eigenvalue problems," SIAM J. Sci. Comput., vol. 24, no. 1, pp. 312-334, July 2002.

[38] J. Money and Q. Ye, "Algorithm 845: EIGIFP: a MATLAB program for solving large symmetric generalized eigenvalue problems," ACM Trans. Math. Softw., vol. 31, pp. 270-279, Jan. 2005.

[39] J. Kiefer, "Sequential minimax search for a maximum," Proc. Am. Math. Soc., vol. 4, no. 3, pp. 502-506, 1953.

[40] Pengfei Xia and G. B. Giannakis, "Design and analysis of transmitbeamforming based on limited-rate feedback," IEEE Trans. Signal Process., vol. 54, no. 5, pp. 1853-1863, 2006.

[41] S. Kutty and D. Sen, "Beamforming for millimeter wave communications: An inclusive survey," IEEE Commun. Surveys Tuts., vol. 18, no. 2, pp. 949-973, 2016.

[42] R. Lenk and C. Lenk, Practical Lighting Design with LEDs. Wiley, 2011.

[43] M. Rea and I. E. S. of North America, The IESNA Lighting Handbook: Reference \& Application. Illuminating Engineering Society of North America, 2000.

[44] Lighting of Indoor Work Places, European Stand. EN 12464-1, European Stand. EN 12464-1, 2003.

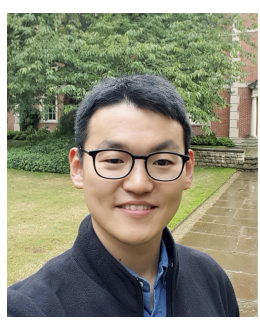

Sunghwan Cho (S'17 - M'21) Sunghwan Cho is an assistant professor at Korea Military Academy, Seoul, South Korea. In 2021, he received his DPhil in Engineering Science from the University of Oxford, U.K. Since 2007, he has worked as an army officer of the Republic of Korea Army, currently holding the rank of a major. His current research interests include visible light communication, physical layer security, electrophoretic molecular communication.

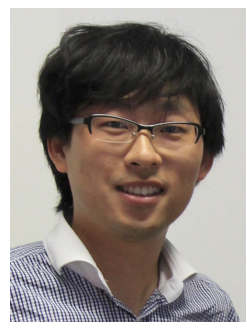

Gaojie Chen (S'09 - M'12 - SM'18) received the B.Eng. and B.Ec. degrees in electrical information engineering and international economics and trade from Northwest University, China, in 2006, and the M.Sc. (Hons.) and Ph.D. degrees in electrical and electronic engineering from Loughborough University, Loughborough, U.K., in 2008 and 2012, respectively. From 2008 to 2009, he was a Software Engineer with DT Mobile, Beijing, China. From 2012 to 2013, he was a Research Associate with the School of Electronic, Electrical and Systems Engineering, Loughborough University. He was a Research Fellow with 5GIC, Faculty of Engineering and Physical Sciences, University of Surrey, U.K., from 2014 to 2015. He was also a Research Associate with the Department of Engineering Science, University of Oxford, U.K., from 2015 to 2018. $\mathrm{He}$ is currently a Lecturer with the School of Engineering, University of Leicester, U.K. His current research interests include information theory, wireless communications, cooperative communications, cognitive radio, the Internet of Things, secrecy communications, and random geometric networks. He received the Exemplary Reviewer Certificates of the IEEE WIRELESS COMMUNICATIONS LETTERS in 2018 and the IEEE TRANSACTIONS ON COMMUNICATIONS in 2019. He serves as an Associate Editor for the IEEE Communications LetTers, IEEE Wireless Communications LETTERS and Electronics Letters (IET).

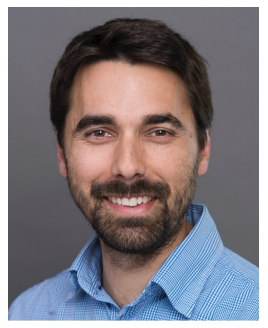

Justin P. Coon (S'02 - M'05 - SM'10) received the B.Sc. degree (Hons.) in electrical engineering from the Calhoun Honours College, Clemson University, Clemson, SC, USA, in 2000, and the Ph.D. degree in communications from the University of Bristol, Bristol, U.K., in 2005. He was with Toshiba Research Europe Ltd., (TREL) from 2004 to 2013. He was also a Visiting Fellow with the School of Mathematics, University of Bristol, from 2010 to 2012. He held a concurrent appointment as a Reader with the Department of Electrical and Electronic Engineering, University of Bristol, from 2012 to 2013. Since 2013, he has been an Associate Professor with the Department of Engineering Science, University of Oxford, Oxford, U.K., and the Emmott Fellow in engineering with the Oriel College, Oxford, where he teaches and conducts research on communication theory, network theory, signal processing for communications, and information theory. He has coauthored more than 180 articles on these topics. He has been an Editor for several IEEE journals. He is also a Fellow of the Institute of Mathematics and Its Applications. 